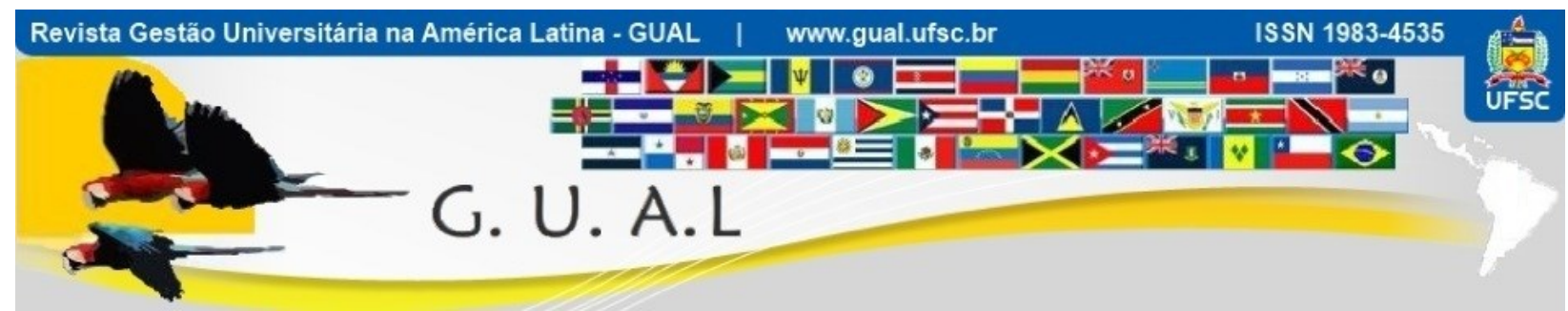

DOI: http://dx.doi.org/10.5007/1983-4535.2018v11n4p57

\title{
AVALIAÇÃO DA EFICIÊNCIA DA ESTRUTURA E DO FUNCIONAMENTO DO SISTEMA DE CONTROLE GERENCIAL COM O PERFORMANCE MANAGEMENT AND CONTROL (PMC) EM DUAS UNIVERSIDADES
}

\author{
EVALUATION OF THE STRUCTURE EFFICIENCY AND PERFORMANCE \\ OF THE PROCESS CONTROL SYSTEM WITH THE PERFORMANCE \\ MANAGEMENT AND CONTROL (PMC) IN TWO UNIVERSITIES
}

Ivonez Xavier de Almeida, Mestre Universidade Comunitária da Região de Chapecó - Unochapecó ivonez.almeida@unochapeco.edu.br

Evanilde Gollo Cordazzo, Mestre Universidade Comunitária da Região de Chapecó - Unochapecó evanilde@unochapeco.edu.br

Rodney Wernke, Doutor Universidade Comunitária da Região de Chapecó - Unochapecó rodneywernke1@hotmail.com

Antonio Zanin, Doutor Universidade Comunitária da Região de Chapecó - Unochapecó zanin@unochapeco.edu.br

Recebido em 24/novembro/2017

Aprovado em 06/setembro/2018

Sistema de Avaliação: Double Blind Review

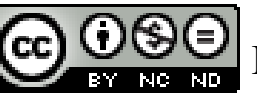

Esta obra está sob uma Licença Creative Commons Atribuição-Uso. 


\title{
AVALIAÇÃO DA EFICIÊNCIA DA ESTRUTURA E DO FUNCIONAMENTO DO SISTEMA DE CONTROLE GERENCIAL COM O PERFORMANCE MANAGEMENT AND CONTROL (PMC) EM DUAS UNIVERSIDADES \\ DOI: http://dx.doi.org/10.5007/1983-4535.2018v11n4p57
}

\section{RESUMO}

Objetivo do estudo foi conhecer a opinião dos coordenadores de curso sobre a utilização dos Sistemas de Controle Gerencial (SCG) para avaliação de desempenho em duas Instituições de Ensino Superior (IES). A metodologia empregada foi descritiva, quantitativa e de levantamento (survey) com base em questionário, que foi enviado a 150 coordenadores de cursos de duas IES, mas retornam apenas 57 respostas (38\%). Na análise de dos dados foi utilizada a estatística descritiva e a entropia da informação para verificar a dispersão nos níveis de escala das respostas coligidas. Os resultados apontaram média de "3,1" (na IES 1) e "3,78" (na IES 2) quanto à concordância nos quesitos gerais do PMC, mostrando que os respondentes concordam parcialmente com a eficiência da estrutura de avaliação de desempenho das duas IES. Porém, acerca do sistema de avaliação de desempenho os resultados evidenciaram divergências entre os contextos das duas entidades pesquisadas. Quanto à eficiência do processo de avaliação as respostas apresentaram dispersão mediana em relação ao conjunto de 16 questões do $\mathrm{PMC}$, se assemelhando a pesquisas anteriores. Concluiu-se pela necessidade de melhoria no alinhamento estratégico dessas entidades, uma vez que os respondentes conhecem o planejamento estratégico, mas há lacunas importantes no desenvolvimento e controle de sua implementação.

Palavras-chave: Avaliação Institucional. Sistemas de Controle Gerencial. Ensino Superior.

\begin{abstract}
The objective of the study was to know the opinion of two course coordinators about the utilization of control systems (SCG) in the performance evaluation in two higher education institution. The methodology utilized was descriptive, quantitative and the survey based in a questionnaire, it was sent to 150 course coordinators of two higher education institutions, with the feedback of 57 answers (38\%). In the data analysis was utilized a descriptive statistics and the entropy of information to verify the grading levels dispersion in associated answers. The results show the average of " 3,1 " in the first higher education institution and " 3,78 " in the second higher education institution and agreement between the PMC, showing that the respondents partially agree with the structure efficiency and performance evaluation in both universities. However, about the performance management system the results confirm divergences between the contexts in the two universities studied. About the evaluation process efficiency the responses showed medium dispersion in relation to the range of 16 questions in PMC, comparing to the earlier researches. It was concluded the need of strategic alignment improvement in this two institutions, once the respondents know the strategic planning, but there are important failures in the development and the control in your implementation.
\end{abstract}

Keywords: Institutional Assessment. Process Control System. Higher Education Institution. Performance Management and Control. 


\section{AVALIAÇÃO DA EFICIÊNCIA DA ESTRUTURA E DO FUNCIONAMENTO DO SISTEMA DE CONTROLE GERENCIAL COM O PERFORMANCE MANAGEMENT AND CONTROL (PMC) EM DUAS UNIVERSIDADES \\ DOI: http://dx.doi.org/10.5007/1983-4535.2018v11n4p57}

\section{INTRODUÇÃO}

No período de 2000 a 2013 houve crescimento de 102,6\% no número de Instituições de Ensino Superior (IES) no Brasil, chegando a 2.391 entidades (MAPA DO ENSINO SUPERIOR, 2015). Esse crescimento do setor educacional mostra sua relevância para a economia do país e pode ser atribuído à necessidade de conhecimento e formação para o mercado de trabalho, exigido dos funcionários pelas empresas que pretendem se manter competitivas em mercados cada vez mais concorridos.

Contudo, esse contexto acarreta um acirramento da competição e as IES são induzidas a adotar novos instrumentos de gestão que facilitem a adaptação ao ambiente de mercado mais concorrido. Com isso, surge a necessidade de promover a modernização dos processos e revisar o modelo de gestão com o fito de aprimorar as suas vantagens competitivas perante os concorrentes (JACOMOSSI; SILVA, 2016). Nesse sentido, os Sistemas Controle Gerencial (SCGs) são fundamentais para medir e gerenciar a performance organizacional, subsidiando especialmente àqueles que desempenham papel estratégico na organização em termos decisoriais (BEUREN; TEIXEIRA, 2014; JACOMOSSI; SILVA, 2016).

Acerca disso, Anthony e Govindarajan (2006) aduzem que para que as organizações funcionarem de maneira que possam atingir seus objetivos é necessário que haja um controle de gestão adequado e os sistemas de controle gerencial são instrumentos que oportunizam e oferecem mecanismos para o controle das estratégias, assegurando que as mesmas sejam obedecidas, de forma que os objetivos sejam atingidos.

Existem vários modelos de controle gerencial, sendo que o Performance Management and Control (PMC) é um modelo adaptado por Ferreira e Otley (2006), a partir dos estudos de Simons (1995) e Otley (1999), cuja configuração mais recente envolvia doze questões. Contudo, Beuren e Teixeira (2014) adaptaram o modelo de Ferreira e Otley (2006) e fizeram um maior detalhamento de alguns pontos específicos, transformando os itens do modelo original em perguntas que, a priori, facilitam a replicação do modelo em várias organizações ou setores.

É pertinente salientar que o PMC sugere uma série de questões a serem abordadas na concepção e funcionamento de um sistema de controle, mas não propõe um “modelo ideal”. É uma ferramenta que pode ser usada para coletar evidências que descrevem as características de um sistema de controle de gestão e as formas de como utilizá-lo no contexto de uma 


\section{AVALIAÇÃO DA EFICIÊNCIA DA ESTRUTURA E DO FUNCIONAMENTO DO SISTEMA DE CONTROLE GERENCIAL COM O PERFORMANCE MANAGEMENT AND CONTROL (PMC) EM DUAS UNIVERSIDADES \\ DOI: http://dx.doi.org/10.5007/1983-4535.2018v11n4p57}

organização. Ainda fornece uma visão geral e a apreciação da estrutura dos sistemas de controle que estão atualmente em uso. Nesse rumo, estudar o desempenho de uma organização é uma tarefa minuciosa e criteriosa devido às inúmeras variáveis e dimensões que envolvem o ambiente organizacional, relacionadas à cultura e a outros fatores organizacionais como estratégia, tamanho, estrutura, tecnologia etc. (FERREIRA; OTLEY, 2006).

Esse diagnóstico acerca dos controles internos pode ser útil também aos gestores das instituições de ensino superior (IES). Destarte, este estudo se propõe a responder à seguinte questão de pesquisa: qual a visão dos coordenadores de curso acerca do uso dos sistemas de controle gerencial (SCG) para avaliação de desempenho em instituições de ensino superior (IES)? Com essa finalidade foi estabelecido o objetivo de conhecer a opinião dos coordenadores de curso sobre a utilização dos SCG para avaliação de desempenho em duas IES comunitárias situadas no oeste de Santa Catarina, por intermédio do Performance Management Control (PMC).

Um estudo com esse foco se justifica pela necessidade de aderência da gestão das entidades universitárias às estratégias institucionais. Isso é pertinente de vez que são os gestores e respectivas equipes de trabalho que devem colocar em prática o que foi estabelecido como missão da universidade por intermédio do cumprimento dos objetivos e das metas da instituição (TACHIZAWA; ANDRADE, 2002). Além disso, pretende-se comparar os resultados obtidos por Beuren e Teixeira (2014) aos achados deste estudo, o que amplia o universo de IES pesquisadas e trará uma dimensão maior sobre a utilização dessa ferramenta para avaliação de desempenho no âmbito desse tipo de entidade.

\section{FUNDAMENTAÇÃO TEÓRICA}

A fim de alcançar seus objetivos as organizações buscam adaptar-se às exigências do mercado e compreender os fenômenos que mais influenciam o ambiente onde atuam. Nesse processo podem ser necessárias reestruturações para manter a conformidade com a visão e a missão (HAMPTON, 1990; BEUREN; SANTANA; THEIS, 2014).

Nessa direção, o planejamento é parte fundamental do processo organizacional, onde o estabelecimento de metas e a definição de estratégias são mecanismos importantes e que subsidiam a tomada de decisões. Com isso, os Sistemas de Controle Gerencial (SCG) são utilizados com o objetivo de assegurar que o comportamento dos funcionários dentro da 


\section{AVALIAÇÃO DA EFICIÊNCIA DA ESTRUTURA E DO FUNCIONAMENTO DO SISTEMA DE CONTROLE GERENCIAL COM O PERFORMANCE MANAGEMENT AND CONTROL (PMC) EM DUAS UNIVERSIDADES \\ DOI: http://dx.doi.org/10.5007/1983-4535.2018v11n4p57}

empresa seja coerente às estratégias e objetivos desta (ANTHONY; GOVINDARAJAN, 2006; MERCHANT; VAN DER STEDE, 2007; BEUREN et al., 2016).

O Sistema de Controle Gerencial (SCG), que na literatura inglesa é denominado de Managment Control Systems (MCS), tem por objetivo obter e utilizar informações com vistas a auxiliar e coordenar as decisões de planejamento e controle organizacional, com a intenção de aprimorar as decisões coletivas (BEUREN; TEIXEIRA; 2014). Acerca disso, Otley (1999) aduz que no SCG os controles são caracterizados pelo uso na gestão e contemplam a mensuração de desempenho e o sistema de recompensas pelo alcance de níveis preestabelecidos. Para o referido autor, os SCGs são sistemas que agrupam informações para utilizá-las na avaliação do desempenho organizacional, podendo ser de algum recurso específico (a exemplo do financeiro) ou da organização como um todo, levando em consideração as estratégias empresariais. Desse modo, os SCGs influenciam o comportamento das entidades em relação às estratégias estabelecidas. Acerca disso, Beuren et al. (2016) asseveram que o SCG afeta os gestores de uma organização, seja pelo desenho ou pela forma de implementação deste.

Os SCGs compreendem procedimentos sistemáticos, oriundos de todas as áreas do conhecimento, desenvolvidos a fim de alcançar os objetivos organizacionais e também contemplam práticas de contabilidade gerencial, a exemplo do orçamento. Dessa forma, compõem os SCGs os controles formais, as informações de rotinas e os procedimentos de gestão (CHENHALL, 2003; DAVILA; FOSTER; LI, 2009; BEUREN; ORO, 2014).

Por seu turno, Jacomossi e Silva (2016) salientam que os SCGs costumam abranger medidas de desempenho não financeiras, fornecendo apoio às decisões estratégicas da instituição. Ainda, de acordo com Beuren e Teixeira (2014), o nível de formalização do controle gerencial pode variar em cada organização, pois é influenciado pelas características de seus administradores, da própria organização e das características do contexto social e organizacional. Por isso, afirmam que os SCGs compreendem procedimentos formais e informais, além dos processos organizacionais, com a finalidade de medir, controlar e gerir o desempenho objetivando definir estratégias alinhadas aos objetivos da empresa. Esclarecem, também, que para serem efetivos os SCGs devem estar alinhados às estratégias da empresa e serem compatíveis em relação à estrutura organizacional e o poder de decisão dos gestores.

O uso de SCG permite elaborar diagnósticos da organização, e a partir destes, aprimorar a gestão. Nisiyama et al. (2016) asseveram que o uso de diagnósticos compreende o 


\section{AVALIAÇÃO DA EFICIÊNCIA DA ESTRUTURA E DO FUNCIONAMENTO DO SISTEMA DE CONTROLE GERENCIAL COM O PERFORMANCE MANAGEMENT AND CONTROL (PMC) EM DUAS UNIVERSIDADES \\ DOI: http://dx.doi.org/10.5007/1983-4535.2018v11n4p57}

monitoramento de variáveis de desempenho e ressaltam que controlar o desempenho é vital para uma empresa, pois este pode ser melhorado a partir da adoção ou alinhamento de estratégias. Esse processo também pode melhorar o processo interno, uma vez que gera aprendizados e em decorrência destes espera-se aprimorar o processo organizacional.

Passos e Spers (2014) afirmam que o sistema de avaliação de desempenho deve abranger todas as variáveis que interferem nos objetivos da empresa. Portanto, deve permitir uma análise global que permita integrar todas as perspectivas da organização. Ou seja, a avaliação do desempenho é parte fundamental para a gestão de uma organização, pois melhorar a performance desta deve ser uma prioridade para os gestores e a adoção de sistema de avaliação facilita atingir esse objetivo (DUTRA, 2003).

Oyodamari et al. (2016) elencam três estilos de avaliação de desempenho utilizadas pelas empresas que foram identificados por Hopwood (1972): (i) a avaliação restrita ao orçamento, na qual o fator determinante é o resultado; (ii) a avaliação com foco de resultado no longo prazo, que se fundamenta na priorização dos resultados de longo prazo e (iii) a avaliação de foco não-contábil, onde o desempenho é avaliado também por indicadores e informações qualitativas, além das informações contábeis. Os mencionados autores ressaltam, também, que o grau de flexibilidade em relação às variações orçamentárias depende de cada empresa, pois num ambiente dinâmico o processo orçamentário não deve ser a única base de avaliação. Nesse sentido, os gestores devem ter liberdade de avaliar o desempenho da organização com base em métricas além das contábeis, pois há indicadores não financeiros que influenciam, fortemente, na tomada de decisão.

Nesse contexto podem ser incluídas as Instituições de Ensino Superior (IES), as quais se diferenciam dos demais tipos de organizações devido à complexidade inerente a aspectos como estrutura, organização e objetivos (SANTOS; BRONNEMAN, 2013). Além disso, na atualidade essas instituições enfrentam diversos desafios, como o acirramento da concorrência, o surgimento de exigências legais mais rígidas e as alterações das normas das entidades reguladoras.

\subsection{PERFORMANCE MANAGEMENT AND CONTROL (PMC)}

Controle é um processo de acompanhar as atividades de uma organização e avaliar sua consistência com o planejamento estratégico para que os objetivos sejam alcançados, assegurando que a organização siga na direção pretendida (ANTHONY; GOVINDARAJAN, 


\section{AVALIAÇÃO DA EFICIÊNCIA DA ESTRUTURA E DO FUNCIONAMENTO DO SISTEMA DE CONTROLE GERENCIAL COM O PERFORMANCE MANAGEMENT AND CONTROL (PMC) EM DUAS UNIVERSIDADES \\ DOI: http://dx.doi.org/10.5007/1983-4535.2018v11n4p57}

2006). O controle gerencial pode ser, então, compreendido como o processo de guiar as organizações em direção a padrões viáveis de atividade em um ambiente caracterizado por mudanças (AGUIAR; FREZATTI, 2007). Para desempenharem essa função de forma satisfatória, precisam do suporte de um sistema de informação, que se constitui em uma rede de informações onde os fluxos alimentam o processo de tomada de decisão, possibilitando a avaliação do desempenho individual ou coletivo e o alinhamento com as estratégias (MOSIMANN; FISCH, 1999; GOMES; SALAS, 2001; RAUPP; MARTINS; BEUREN, 2006). Destarte, para se obter uma avaliação de desempenho o mais coerente possível, é necessário que se tenha uma estrutura de gerenciamento de desempenho e controle adequada. Para cumprir com seu papel organizacional um sistema de controle gerencial necessita de informações para manter ou alterar padrões nas atividades cotidianas que são obtidas por meio de diferentes mecanismos de controle (SIMONS, 1995; DRURY, 2004).

Nessa direção, Simons (1995) desenvolveu um sistema estruturado de controle gerencial que visa equilibrar tensões decorrentes das necessidades de controle, o que é típico de ambientes competitivos. Os constructos-chave se baseiam no núcleo de valores, nos riscos a serem evitados, nas incertezas estratégicas e nas variáveis de desempenho críticas, que são operacionalizados e controlados através de quatro sistemas ou alavancas de controle: Sistemas de Crenças; Sistemas de Limites (ou Restrições); Sistemas de Controle Diagnóstico e Sistemas de Controle Interativo.

Entretanto, aspectos positivos e negativos, estimulantes e restritivos fazem parte dos controles de gestão e as organizações precisam balancear seu uso ao longo do tempo. Simons (1995) sugere que o Sistema de Crenças e o Sistema Interativo criam energias positivas, enquanto o Sistema de Diagnóstico e o Sistema de Restrições criam energias negativas, cujas forças opostas atuam em equilíbrio, criando uma interdependência e um processo de causa e efeito.

Por outro prisma, a estrutura do sistema de controle gerencial proposto por Otley (1999) é integrada por cinco questões centrais relacionadas à gestão moderna e abordam: (i) a definição de objetivos fundamentais e avaliação da realização dos objetivos; (ii) estratégias e planos organizacionais e processo de medição e avaliação de implementação; (iii) definição de metas e avaliação do cumprimento das mesmas; (iv) sistema de recompensas (ou punições) a serem estabelecidas e (v) tipos de fluxo de informações (feedback e feedforward) 


\section{AVALIAÇÃO DA EFICIÊNCIA DA ESTRUTURA E DO FUNCIONAMENTO DO SISTEMA DE CONTROLE GERENCIAL COM O PERFORMANCE MANAGEMENT AND CONTROL (PMC) EM DUAS UNIVERSIDADES \\ DOI: http://dx.doi.org/10.5007/1983-4535.2018v11n4p57}

necessários para acompanhar o desempenho, além de realizar e manter a aprendizagem organizacional.

A estrutura de Gerenciamento de Desempenho e Controle (Performance Management and Control $-P M C$ ) é um instrumento proposto por Ferreira e Otley (2006), que busca captar a estrutura e o funcionamento dos Sistemas de Controle Gerencial (SGC). Os mencionados autores tomaram como referência os desenhos anteriores de Simons (1995) e Ottley (1999), ampliando o modelo para doze questões com a finalidade de fornecer um instrumento mais útil àqueles que estudam o projeto e a operacionalização dos sistemas de controle gerencial e desempenho. Caracteriza-se por ser uma estrutura holística que visa analisar, de forma ampla, a gestão de desempenho.

Essa estrutura proposta por Ferreira e Otley (2006) procura especificar os aspectos da gestão relacionados à visão, à missão e à forma de transmissão destas na organização; aos principais fatores de sucesso; à estrutura organizacional e o impacto desta na concepção e gestão de sistema de desempenho; às estratégias e planos adotados, bem como processos e atividades consideradas necessárias para o sucesso da organização; às principais medidas de desempenho decorrentes dos objetivos; aos fatores críticos de sucesso e estratégias; ao nível de desempenho (metas estabelecidas); aos processos de avaliação dos indivíduos, dos grupos e da organização; aos sistemas de recompensa (ou de punição); aos fluxos de informações, bem como ao uso de informação e aos mecanismos de controle. Além disso, determinadas questões (Q11 e Q12) querem demonstrar como os sistemas de gestão do desempenho têm sido alterados em função das mudanças dinâmicas da organização e seu ambiente, e quão forte e coerente são as ligações entre os componentes do sistema de gerenciamento do desempenho. A estrutura pretende colaborar para o entendimento de como o processo de gestão do desempenho ocorre nas organizações envolvendo planejamento, análise de resultado e retroalimentação (FERREIRA; OTLEY, 2006).

\subsection{ESTUDOS ASSEMELHADOS}

Algumas pesquisas anteriores abordaram a avaliação dos sistemas de controle gerencial em empresas industriais/comerciais brasileiras (DAL MAGRO; GORLA; LAVARDA, 2014; PASSOS; SPEARS, 2014; BORTOLUZZI; ENSSLIN; ENSSLIN, 2015). Contudo, em instituições de ensino superior, somente Beuren e Teixeira (2014) aplicaram o modelo PMC com o objetivo de averiguar a estrutura e o funcionamento dos sistemas de 


\section{AVALIAÇÃO DA EFICIÊNCIA DA ESTRUTURA E DO FUNCIONAMENTO DO SISTEMA DE CONTROLE GERENCIAL COM O PERFORMANCE MANAGEMENT AND CONTROL (PMC) EM DUAS UNIVERSIDADES \\ DOI: http://dx.doi.org/10.5007/1983-4535.2018v11n4p57}

controle gerencial para avaliação de desempenho em uma entidade privada. Os resultados apontaram para a necessidade de um melhor alinhamento entre a estratégia empresarial, o sistema de avaliação de desempenho e os sistemas de controle gerencial no contexto daquela entidade.

\section{METODOLOGIA}

Este estudo caracteriza-se como "descritivo", visto que o objetivo principal deste tipo de pesquisa é descrever as características de determinada população ou fenômeno, estabelecendo relações entre as variáveis (SILVA, 2003). Quanto a abordagem é quantitativa, pois utiliza-se de quantificação e métodos estatísticos para o tratamento e a análise dos dados (RICHARDSON, 2014). No que tange aos procedimentos, classifica-se como levantamento, de vez que essa modalidade de estudo visa obter informações sobre uma determinada população (ROESCH, 2006). Para efetuar tal levantamento foi utilizado um questionário específico, fundamentado no instrumento Performance Management and Control (PMC) proposto por Ferreira e Otley (2006), posteriormente adaptado por Beuren e Teixeira (2014), que ampliaram-no para dezesseis perguntas. Contudo, na pesquisa ora relatada tal questionário foi ampliado para vinte e uma questões, divididas em duas seções.

A primeira parte compreende as perguntas de um a cinco e referem-se ao perfil do respondente, enquanto que a segunda seção é composta pelas questões " 6 " a "21", que estão efetivamente relacionadas ao Performance Management and Control (PMC). Nesta última parte, todas as perguntas têm o formato de múltipla escolha como opção de respostas. Para tanto, utiliza-se uma escala do tipo Likert de cinco pontos, em que os níveis "1" e "5" significam "discordo totalmente" e "concordo totalmente", respectivamente. Ainda, os graus "2" e "4" significam discordância e concordância parcial e o patamar "3" representa "nem discordo/nem concordo".

Para validar as adaptações efetuadas no questionário, este foi enviado a um coordenador de curso de outra instituição de ensino superior (não participante do estudo), o qual não sugeriu qualquer alteração, considerando-o de fácil entendimento. Posterior a isso o questionário foi enviado a 150 (cento e cinquenta) coordenadores e coordenadores adjuntos de cursos das duas instituições priorizadas por meio de correio eletrônico, acompanhado de texto explicativo sobre o objetivo da pesquisa, confidencialidade e finalidade dos dados. Nesse sentido, o e-mail enviado indicou o link eletrônico para acesso direto ao questionário online 


\section{AVALIAÇÃO DA EFICIÊNCIA DA ESTRUTURA E DO FUNCIONAMENTO DO SISTEMA DE CONTROLE GERENCIAL COM O PERFORMANCE MANAGEMENT AND CONTROL (PMC) EM DUAS UNIVERSIDADES \\ DOI: http://dx.doi.org/10.5007/1983-4535.2018v11n4p57}

da pesquisa, valendo-se da ferramenta Google Docs. Assim, após quatro rodadas de envio nos meses de novembro a dezembro de 2016 foram obtidas 57 (cinquenta e sete) respostas, representando $38 \%$ do número total possível.

A escolha das duas instituições onde foi efetuado o estudo se deu pelo critério de acessibilidade dos respondentes e, por solicitação dos administradores, estas não serão identificadas neste relato e serão designadas apenas por IES 1 e IES 2. Contudo, ambas estão situadas no estado de Santa Catarina e, juntas, possuem sete campis onde são ofertados quase uma centena de cursos (graduação, tecnólogos, presencial e a distância, especialização, mestrado e doutorado) em várias áreas do conhecimento.

Para a análise dos dados, utilizou-se da estatística descritiva para obter, principalmente, a média das respostas. Entretanto, nos dados relacionados às questões "6" a “21" do questionário utilizado efetuou-se também a análise da dispersão nos níveis de escala da resposta por meio do cálculo da entropia da informação. A entropia é considerada uma medida simples, porém importante, devido à quantidade de informações fornecida pela mesma (ZELENY, 1982). Sobre essa ferramenta, Rocha (2010) explica o cálculo da entropia informacional como exposto a seguir. Sejam $d_{i}=\left(d_{i}^{1}, d_{i}^{2}, \ldots, d_{i}^{m}\right)$ os valores normalizados, em que: $d_{i}^{k}=\frac{x_{i}^{k}}{x_{i}}$, caracteriza o conjunto D, em termos do i-ésimo atributo. Encontra-se $D_{i}=\sum_{k=1}^{m} d_{i}^{k} ; i=1,2, \ldots, n$. Busca-se então a medida de entropia do contraste de intensidade para o i-ésimo atributo calculado por $e\left(d_{i}\right)=-\alpha \sum_{k=1}^{m} \frac{d_{i}^{k}}{D_{i}} \operatorname{Ln}\left(\frac{d_{i}^{k}}{D_{i}}\right)$, em que $\alpha=\frac{1}{\theta_{\max }}>0$ e $\mathrm{e}_{\max }=\mathrm{Ln}(\mathrm{m})$. Observa-se ainda que $0 \leq d_{i}^{k} \leq 1$ e $d_{i}^{k} \geq 0$. Caso todos os $d_{i}^{k}$ forem iguais para um dado i, então $\frac{d_{i}^{k}}{D_{i}}=\frac{1}{n} \mathrm{e} e\left(\mathrm{~d}_{\mathrm{i}}\right)$ assume o valor máximo, isto é, $\mathrm{e}_{\max }=\operatorname{Ln}(\mathrm{m})$. Ao se fixar $\alpha=\frac{1}{\theta_{\max }}$, determina-se $0 \leq e\left(d_{i}\right) \leq 1$ para todos os $\mathrm{e}\left(\mathrm{d}_{\mathrm{i}}\right) \mathrm{s}$, sendo que essa normalização é necessária para efeito comparativo. Dessa maneira, a entropia total de D é definida por: $E=\sum_{i=1}^{n} e\left(d_{i}\right)$. Quanto maior for $\mathrm{e}\left(\mathrm{d}_{\mathrm{i}}\right)$, menor é a informação transmitida pelo i-ésimo atributo. Caso $\mathrm{e}\left(\mathrm{d}_{\mathrm{i}}\right)=\mathrm{e}_{\max }=\operatorname{Ln}(\mathrm{m})$, então o i-ésimo atributo não transmite informação e pode ser removida da análise decisória. Em virtude do peso $\lambda_{i}$ ser inversamente relacionado a e $\left(\mathrm{d}_{\mathrm{i}}\right)$,

usa-se $1-\mathrm{e}\left(\mathrm{d}_{\mathrm{i}}\right)$ ao invés de $\mathrm{e}\left(\mathrm{d}_{\mathrm{i}}\right)$ e normaliza-se para assegurar que $0 \leq \tilde{\lambda}_{i} \leq 1$ e $\sum_{i=1}^{n} \tilde{\lambda}_{i}=1$. Assim, a entropia da informação pode ser representada por: 


\section{AVALIAÇÃO DA EFICIÊNCIA DA ESTRUTURA E DO FUNCIONAMENTO DO SISTEMA DE CONTROLE GERENCIAL COM O PERFORMANCE MANAGEMENT AND CONTROL (PMC) EM DUAS UNIVERSIDADES \\ DOI: http://dx.doi.org/10.5007/1983-4535.2018v11n4p57}

$$
\tilde{\lambda}_{i}=\frac{1}{n-E}\left[1-e\left(d_{i}\right)\right]=\frac{\left[1-\theta\left(d_{i}\right)\right]}{n-E} .
$$

\section{ANÁLISE DOS RESULTADOS}

O questionário aplicado visava na primeira parte conhecer o perfil dos profissionais abrangidos. Nesse sentido, na questão inicial o participante deveria indicar sua função no curso, onde se constatou que 55 dos 57 respondentes eram coordenadores de curso $(96,5 \%$ do total), onde "13" pertencem à IES 1 e “42” à IES 2, apenas dois eram coordenadores adjuntos (vinculados a IES 1).

Em seguida, se procurou identificar os cursos que os respondentes coordenavam e os dados coligidos permitiram concluir que quatorze coordenadores pertenciam aos cursos de Administração e Ciências Contábeis (sete em cada curso); quatro trabalhavam no curso de Educação Física; nos cursos de Agronomia, Direito e Psicologia participaram três coordenadores de cada curso; Arquitetura \& Urbanismo, Ciências Biológicas e Pedagogia contavam com dois respondentes para cada curso e apenas um participante para cada um dos cursos de Bacharel e Licenciatura, Biotecnologia Industrial e Engenharia de Alimentos, Ciências da Computação, Ciências Econômicas, Comunicação, Coordenação Administrativa, Cosmetologia e Estética, Cursos EAD de C. Contábeis e Tec. em Processos Gerenciais, Design, Design e Jogos Digitais, Enfermagem, Engenharia Civil, Engenharia de Alimentos, Engenharia Mecânica, Engenharia Química, Engenharia Sanitária e Ambiental, Fisioterapia, Letras, Licenciatura em Química, Pedagogia e Música, Medicina Veterinária, Odontologia, Serviço Social, Tecnólogo em Análise e Desenvolvimento de Sistemas e Tecnólogo em Gestão de RH.

$\mathrm{Na}$ sequência foi inquirido sobre o período de tempo que os respondentes estão exercendo a função mencionada. Nesse rumo, apurou-se que vinte dos respondentes estão na função há menos de dois anos $(35,09 \%$ do total) e quinze estão na função entre dois e quatro anos $(26,32 \%)$. Ainda, dez participantes do estudo estão no cargo atual entre quatro e seis anos (17,54\%); enquanto que apenas dois estão na função entre seis e oito anos e dez há mais de oito anos $(3,51 \%$ e $17,54 \%$, respectivamente).

A quarta questão pretendeu conhecer o tempo de trabalho na instituição, abrangendo também as demais funções exercidas. Acerca disso, apenas um respondente afirmou que atua na instituição há menos de dois anos (1,75\%), quatorze estão vinculados à instituição entre dois e cinco anos $(24,56 \%)$ e doze têm vínculo por prazo entre cinco e dez anos $(21,05 \%)$. 
Ainda, onze trabalham na IES entre dez e quinze anos (19,30\%) e dezenove atuam há mais de 15 anos (33,33\%). Os prazos dilatados mencionados podem indicar que as duas universidades mantêm algum programa ou forma incentivo visando a retenção de talentos, pois mais da metade dos respondentes $(52,63 \%)$ trabalham nelas há mais de 10 anos.

A pergunta "5" almejava evidenciar a titulação dos respondentes, onde os dados coligidos denotam que 45 dos 57 respondentes é mestre (78,95\%), dez são doutores $(17,54 \%)$ e somente dois $(3,51 \%)$ possuem título de especialista. Esses percentuais sugerem que os profissionais buscaram formação mais aprimorada a fim de qualificar-se para a função de coordenadores, o que favorece as IES no sentido de melhorar sua avaliação pelos órgãos competentes pelo número de profissionais devidamente qualificados.

\subsection{ANÁLISE DESCRITIVA DAS QUESTÕES RELACIONADAS AO PMC}

Nesta seção são apresentados e comentados os resultados amealhados em relação às perguntas "6" a " 21 " do instrumento utilizado. Para tanto, foi adotado uma escala do tipo Likert nas opções de respostas do questionário apresentado aos participantes que abrangia "Concordo Totalmente", "Concordo Parcialmente", "Não Concordo/Nem Discordo", "Discordo Parcialmente" e "Discordo Totalmente". Ainda é pertinente esclarecer que os dados foram analisados em três blocos correspondentes a (i) estratégia (Q6 a Q9), (ii) sistema de avaliação de desempenho (Q10 a Q15) e (iii) controle de gestão (Q16 a Q21). Assim, na Tabela 1 estão sumarizados os dados coletados, evidenciando para todas as questões o percentual de participação por IES.

Tabela 1 Análise descritiva

\begin{tabular}{c|c|c|c|c|c|c|c|c|c|c}
\hline \multirow{2}{*}{ Questões } & \multicolumn{9}{|c|}{ IES 1 } & \multicolumn{6}{c}{ IES 2 } \\
\cline { 2 - 10 } & $\begin{array}{c}\mathrm{CT} \\
\%\end{array}$ & $\begin{array}{c}\mathrm{CP} \\
\%\end{array}$ & $\begin{array}{c}\mathrm{NC} / \mathrm{ND} \\
\%\end{array}$ & $\begin{array}{c}\mathrm{DP} \\
\%\end{array}$ & $\begin{array}{c}\mathrm{DT} \\
\%\end{array}$ & $\begin{array}{c}\mathrm{CT} \\
\%\end{array}$ & $\begin{array}{c}\mathrm{CP} \\
\%\end{array}$ & $\begin{array}{c}\text { NC/ND } \\
\%\end{array}$ & $\begin{array}{c}\text { DP } \\
\%\end{array}$ & $\begin{array}{c}\text { DT } \\
\%\end{array}$ \\
\hline 6 & 26,67 & 60,00 & - & 13,33 & - & 57,14 & 35,71 & 2,38 & 2,38 & 2,38 \\
7 & 13,33 & 73,33 & - & 13,33 & - & 50,00 & 40,48 & 4,76 & 2,38 & 2,38 \\
8 & 6,67 & 53,33 & 13,33 & 26,67 & - & 54,76 & 30,95 & 7,14 & 7,14 & - \\
9 & - & 13,33 & 20,00 & 26,67 & 40,00 & 45,24 & 26,19 & 7,14 & 11,90 & 9,52 \\
10 & 13,33 & 26,67 & 40,00 & 20,00 & - & 66,67 & 23,81 & 2,38 & 7,14 & - \\
11 & - & 46,67 & 33,33 & 20,00 & - & 45,24 & 40,48 & 4,76 & 9,52 & - \\
12 & 6,67 & 46,67 & 26,67 & 20,00 & - & 23,81 & 54,76 & 9,52 & 9,52 & 2,38 \\
13 & - & 46,67 & 26,67 & 13,33 & 13,33 & 19,05 & 52,38 & 9,52 & 16,67 & 2,38 \\
14 & - & 26,67 & 33,33 & 26,67 & 13,33 & 11,90 & 30,95 & 19,05 & 11,90 & 26,19 \\
15 & - & 20,00 & 46,67 & 26,67 & 6,67 & 7,14 & 28,57 & 11,90 & 16,67 & 35,71
\end{tabular}




\section{AVALIAÇÃO DA EFICIÊNCIA DA ESTRUTURA E DO FUNCIONAMENTO DO SISTEMA DE CONTROLE GERENCIAL COM O PERFORMANCE MANAGEMENT AND CONTROL (PMC) EM DUAS UNIVERSIDADES \\ DOI: http://dx.doi.org/10.5007/1983-4535.2018v11n4p57}

\begin{tabular}{c|c|c|c|c|c|c|c|c|c|c}
\hline & & & & & & & & & \\
16 & - & 33,33 & 13,33 & 20,00 & 33,33 & 38,10 & 33,33 & 2,38 & 9,52 & 16,67 \\
17 & 6,67 & 33,33 & 13,33 & 20,00 & 26,67 & 33,33 & 28,57 & 9,52 & 16,67 & 11,90 \\
18 & - & 26,67 & 20,00 & 20,00 & 33,33 & 23,81 & 33,33 & 11,90 & 16,67 & 14,29 \\
19 & 13,33 & 40,00 & 33,33 & 6,67 & 6,67 & 16,67 & 50,00 & 21,43 & 9,52 & 2,38 \\
20 & 20,00 & 33,33 & 26,67 & 20,00 & - & 19,05 & 54,76 & 14,29 & 9,52 & 2,38 \\
21 & - & 40,00 & 33,33 & 26,67 & - & 30,95 & 38,10 & 14,29 & 16,67 & - \\
\hline
\end{tabular}

Fonte: dados de pesquisa.

Como mencionado, as questões "6" a "9" abordavam as estratégias adotadas pelas IES. Na primeira pergunta (Q6) foi apurado sobre o conhecimento de aspectos como missão e visão. Na IES 1 cerca de $26,67 \%$ dos respondentes concordaram totalmente e $60 \%$ parcialmente, enquanto que $13,33 \%$ aduziram que discordam parcialmente. Por sua vez, na IES 2 o maior percentual $(57,14 \%)$ correspondeu à resposta "concordo totalmente" e 35,71\% à "concordo parcialmente" (o que totaliza $92,85 \%$ e demonstra boa disseminação do plano estratégico).

Em relação aos objetivos estratégicos que sustentam o futuro da instituição, retratados na questão "7”, estes são totalmente conhecidos por 13,33\% dos respondentes e parcialmente por $73,33 \%$ na IES 1 , mas $13,33 \%$ dos respondentes discordam parcialmente. Na IES 2 foi apurado que $50 \%$ dos participantes concordam totalmente e $40,48 \%$ parcialmente. Ou seja, $90,48 \%$ responderam positivamente sobre este aspecto.

A implementação do planejamento estratégico se dá por meio de processos e atividades e conhecê-los permite uma aproximação entre a descrição e a execução. Questionados sobre este conhecimento (pergunta "8”), na IES 1 apenas 6,67\% concordaram totalmente, outros 53,33\% concordaram de forma parcial e $40 \%$ discordaram parcialmente ou alegaram indiferença. Na IES 2, houve concordância total ou parcial por 85,71\% respondentes, o que mostra um percentual significativo de concordantes nesta entidade.

E relação à questão "9", que trata do recebimento formal do planejamento estratégico diretamente da alta administração, na IES 1 se constatou que 40\% discordaram totalmente e $46,67 \%$ discordaram parcialmente ou mantiveram-se com posição nula. Na IES 2, em torno de $71,43 \%$ concordaram parcial ou totalmente, demonstrando um grande alinhamento na disseminação do planejamento estratégico. Portanto, infere-se a partir dos dados levantados que na IES 1 há uma lacuna entre a elaboração e a disseminação para execução do planejamento estratégico, visto que não foi adequadamente divulgado a todos os coordenadores de cursos. 


\section{AVALIAÇÃO DA EFICIÊNCIA DA ESTRUTURA E DO FUNCIONAMENTO DO SISTEMA DE CONTROLE GERENCIAL COM O PERFORMANCE MANAGEMENT AND CONTROL (PMC) EM DUAS UNIVERSIDADES \\ DOI: http://dx.doi.org/10.5007/1983-4535.2018v11n4p57}

Os resultados apurados em relação a planejamento estratégico (Q6 a Q9) demonstram cenários distintos. Na IES 1 os maiores percentuais de respostas são de profissionais que concordam parcialmente, apresentando também grande concentração nos itens de discordância. Na IES 2 o maior percentual está concentrado em concordo totalmente, com baixa representação dos itens de discordância. Quando comparados ao estudo de Beuren e Teixeira (2014), os resultados da IES 2 são assemelhados, enquanto que da IES 1 são divergentes tendo em vista que evidenciou limitações quanto à disseminação à totalidade dos gestores.

O segundo grupo de perguntas desta parte abordou o sistema de avaliação de desempenho, sendo composto pelas questões "10" a "15". A indagação inicial (Q10) tratava da estrutura existente para adotar o sistema de avaliação de desempenho que influencia ou é influenciado pelo planejamento estratégico. Na IES 1 aproximadamente $60 \%$ dos respondentes discordaram parcialmente ou não se posicionaram e $40 \%$ concordaram total ou parcialmente. Posição inversa foi encontrada na IES 2, quando 90,48\% concordaram total ou parcialmente a respeito deste tema.

No que concerne à adoção de indicadores-chave ligados aos objetivos estratégicos (Q11), constatou-se que na IES 1 houve $46,67 \%$ de respostas que concordaram parcialmente, $33,33 \%$ ficaram neutros e $20 \%$ discordaram parcialmente. Novamente na IES 2 o percentual de concordantes é significativamente maior, alcançando $85,72 \%$ do total.

O estabelecimento de metas para atingimento dos resultados do planejamento estratégico é necessário para todas as organizações e foi o foco da questão "12". Na IES 1 em torno de $46,67 \%$ dos participantes concordaram parcialmente, sendo que 46,67\% se mantém em posição neutra ou discordaram parcialmente. Na IES 2 se apurou que 54,76\% concordaram parcialmente e $23,81 \%$ totalmente, garantindo $78,57 \%$ de anuência.

No que tange ao processo de avaliação de desempenho individual ou coletivo (áreas de ensino, cursos e instituição), este foi o tema abordado na questão "13" onde a IES 1 apresentou percentual de concordância de 46,67\%, mas as posições de discordância ou de nulidade chegaram a 53,33\%. Na IES 2 foi identificado que 52,38\% concordaram parcialmente e $19,05 \%$ totalmente, representando $71,43 \%$ do total de respondentes.

As recompensas ofertadas pelo processo de avaliação de desempenho existente (tema da questão "14") foram consideradas total ou parcialmente justas e ponderadas por $26,67 \%$ 


\section{AVALIAÇÃO DA EFICIÊNCIA DA ESTRUTURA E DO FUNCIONAMENTO DO SISTEMA DE CONTROLE GERENCIAL COM O PERFORMANCE MANAGEMENT AND CONTROL (PMC) EM DUAS UNIVERSIDADES \\ DOI: http://dx.doi.org/10.5007/1983-4535.2018v11n4p57}

dos respondentes da IES 1 e 42,85\% da IES 2. Portanto, nas duas IES pesquisadas há indícios do não reconhecimento da oferta de recompensa pela performance dos gestores.

Por sua vez, a questão "15" indagava sobre a possibilidade de penalização (financeira ou não) no caso das metas propostas não serem atingidas. No que concerne à IES 1, esta apresentou $46,67 \%$ de respondentes que optaram pela posição nula/neutra, enquanto que houve 33,34\% de discordância. No caso da IES 2, os posicionamentos de nulidade e de discordância somados atingiram $64,28 \%$ dos participantes. Com isso, há certa semelhança entre os desempenhos das duas entidades neste quesito.

Tomadas em conjunto, as respostas às cinco questões relativas ao sistema de avaliação de desempenho demonstraram que há necessidade de ajustes no processo, uma vez que parcela significativa dos gestores deixou evidente que não reconhece (ou desconhece) a vinculação entre indicadores de desempenho e a visão estratégica das instituições. Isso ocorreu principalmente na IES 1, cuja posição dos respondentes se concentrou como nula/neutra (nem concordo, nem discordo), além de ter parcela significativa como discordantes. Na IES 2, apesar de ter maior parcela de respondentes que concordam total ou parcialmente, existem lacunas que devem ser avaliadas. Nesse sentido, a percepção de justiça (penalização) pode interferir positiva ou negativamente no desempenho individual ou no do grupo, dependendo dos acordos firmados pela administração. Além disso, ao cotejar esse cenário com os dois estudos anteriores utilizados como referência (FERREIRA; OTLEY, 2006; BEUREN; TEIXEIRA, 2014), percebeu-se que os achados daquelas pesquisas são semelhantes às respostas aqui encontradas.

O último grupo a ser analisado envolvia as questões " 16 " a " 21 ", que se referem ao controle de gestão para avaliação de desempenho. Sobre o recebimento de informações (por escrito ou verbais) acerca do acompanhamento da avaliação do desempenho do gestor (pergunta “16"), as respostas da IES 1 deram conta que 33,33\% aquiesceram parcialmente a respeito. Mas, um contingente expressivo revelou que há falhas nesse aspecto, pois 33,33\% discordaram totalmente, $20 \%$ discordaram parcialmente e 13,33\% ficaram em dúvida sobre o tema. Já para a IES 2 esse tema obteve a concordância de 71,43\% dos participantes e apenas $26,19 \%$ discordaram total ou parcialmente.

Por seu turno, a questão "17" abordava sobre o recebimento de informações do tipo diagnóstico (feedback) que possibilitassem ao gestor avaliar seu próprio desempenho. Os gestores da IES 1 apresentaram maior tendência de discordância e ou de posicionamento 


\section{AVALIAÇÃO DA EFICIÊNCIA DA ESTRUTURA E DO FUNCIONAMENTO DO SISTEMA DE CONTROLE GERENCIAL COM O PERFORMANCE MANAGEMENT AND CONTROL (PMC) EM DUAS UNIVERSIDADES \\ DOI: http://dx.doi.org/10.5007/1983-4535.2018v11n4p57}

neutro, representando $60 \%$ do total, enquanto que $40 \%$ concordavam parcial ou totalmente. A mesma questão conseguiu nível de concordância parcial ou total com $61,9 \%$ dos gestores da IES 2. Ainda assim, determinados participantes discordaram totalmente (11,9\%), outros parcialmente $(9,52 \%)$ e alguns mantiveram-se indiferentes $(9,52 \%)$.

A pergunta "18" inquiria a respeito do fornecimento de informes interativos (também conhecidos como feedforward) que permitem avaliar o desempenho do coordenador de curso durante o período, o que poderia facilitar a correção de rumo visando atingir as metas antes do encerramento prazo estabelecido. Nesse rumo, 33,33\% dos participantes da IES 1 responderam discordar totalmente, 20\% alegaram discordar parcialmente e 20\% mantiveramse indiferentes/neutros. Portanto, apenas $26,67 \%$ dos gestores dessa entidade concordaram parcialmente. A mesma questão é de concordância total ou parcial para 57,14\% dos gestores da IES 2. Entretanto, apesar de conseguir um patamar melhor em relação à IES 1, ainda assim há parcela substancial de gestores $(30,97 \%)$ que discordaram total ou parcialmente e 11,9\% mantiveram-se indiferentes sobre isso.

A questão "19" tratava sobre a hipótese de que a dinâmica das atividades da organização e do ambiente onde atua possam influenciar na avaliação de desempenho e esta ser alterada pelos referidos fatores. Os dados coligidos indicaram que houve concordância plena a respeito por $13,33 \%$ dos respondentes, parcial por $40 \%$ e indiferente por $33,33 \%$ dos participantes da IES 1. Os respondentes da IES 2, por sua vez, concordaram totalmente em $16,67 \%$ das respostas desta questão e parcialmente em $50 \%$ dos casos. Contudo, $21,43 \%$ se manifestaram como indiferentes e $11,9 \%$ aduziram que discordam parcial ou totalmente. Embora as IES apresentem percentuais diferentes, alinham-se nos percentuais de concordância parcial e de indiferença/neutralidade.

A pergunta " 20 " indagava se já havia ocorrido alterações na avaliação de desempenho dos coordenadores de curso em razão da dinâmica e do ambiente, conforme mencionado no parágrafo precedente. As respostas evidenciaram que isso já ocorreu na opinião de $20 \%$ dos inquiridos da IES 1, mas 33,33\% concordaram apenas parcialmente e 26,67\% mantiveram-se indiferentes, enquanto que $20 \%$ discordaram parcialmente. Na IES 2 restou evidenciado que $73,81 \%$ concordaram total ou parcialmente a respeito, enquanto que uma parcela de $14,29 \%$ dos participantes se mantiveram indiferentes e 11,9\% discordaram parcial ou totalmente.

A última questão " 21 " pretendia colher a opinião sobre a força e a coerência dos procedimentos adotados pela entidade para gerenciar, controlar e avaliar o desempenho. A 


\section{AVALIAÇÃO DA EFICIÊNCIA DA ESTRUTURA E DO FUNCIONAMENTO DO SISTEMA DE CONTROLE GERENCIAL COM O PERFORMANCE MANAGEMENT AND CONTROL (PMC) EM DUAS UNIVERSIDADES \\ DOI: http://dx.doi.org/10.5007/1983-4535.2018v11n4p57}

partir dos dados apurados se percebeu que na IES 1 houve concordância parcial por $40 \%$ dos respondentes, enquanto que $33,33 \%$ se mantiveram indiferentes e $26,67 \%$ discordaram parcialmente. Na IES 2 as respostas apontaram para concordância total de 30,95\% dos respondentes e em $38,10 \%$ destes há concordância parcial. Um contingente menos expressivo se manteve indiferente $(14,29 \%)$ e $16,67 \%$ discordaram parcialmente.

O conjunto de respostas sobre os controles de gestão de desempenho indicam limitações na devolutiva de informações, principalmente na IES 1, quando parcela significativa dos respondentes se mantiveram indiferentes ou citaram ser parcialmente discordantes. A IES 2 apresentou índice de concordância superior à IES 1, revelando haver alinhamento maior, tanto nas informações de feedback como as de feedforward. As respostas desse bloco se coadunam com as pesquisas de Ferreira e Otley (2006) e Beuren e Teixeira (2014), que revelaram índices semelhantes de respostas nos contextos que abrangeram.

Ao analisar os percentuais de respostas das duas IES para o conjunto completo de questões dessume-se que os respondentes da IES 1 estão mais propensos a manterem-se indiferentes/neutros (não concordam, nem discordam) e a discordarem de forma parcial ou total, em relação às questões estratégicas, ao controle e ao sistema de avaliação de desempenho. Já no âmbito da IES 2 apenas duas questões (Q14 e Q15) apresentaram índices de concordância (total ou parcial) inferiores a 50\%, indicando maior alinhamento das respostas. Ainda assim, parcela importante de respondentes apontou para a necessidade de ajustes em relação ao controle de gestão, haja vista que nas questões Q14 a Q21 os índices de indiferença ou de não concordância foram maiores que as questões antecedentes.

É pertinente ressaltar também que dois componentes importantes na avaliação de desempenho - recompensa e punição - são vistos da mesma forma nas duas instituições. A priori, as respostas apontam para a falta de uma política de recompensa e punição, o que é necessário para o alcance das metas individuais e coletivas. Para Reis Neto e Marques (2004), a implementação de sistemas de remuneração alinhados à estratégia, à estrutura, aos processos e às pessoas é uma base essencial para a construção de uma organização eficaz.

\subsection{ENTROPIA DA INFORMAÇÃO}

Os resultados das questões relacionadas ao PMC também foram submetidos à análise da entropia da informação, por meio do cálculo desta e da média respectiva. O objetivo dessa 
iniciativa é identificar as questões que apresentaram maior variabilidade nas respostas, o que pode indicar falta de alinhamento por parte dos coordenadores, na respectiva questão.

Como citado anteriormente, a "entropia $\mathrm{e}\left(\mathrm{d}_{\mathrm{i}}\right)$ " representa o valor informacional do $i$ ésimo atributo (questão), enquanto que o "peso" representa o valor da entropia dessa informação, sendo que quanto maior a entropia, maior a dispersão dos níveis de respostas na escala. Por sua vez, a "média" indica o ponto na escala Likert no qual se concentrou a média das respostas dadas a cada pergunta. Referidos aspectos estão expostos na Tabela 2, apresentada na sequência.

Tabela 2 Análise da entropia da informação

\begin{tabular}{c|c|c|c|c|c|c}
\hline \multirow{2}{*}{ Questões } & \multicolumn{3}{|c|}{ IES 1 } & \multicolumn{2}{c}{ IES 2} \\
\cline { 2 - 7 } & $\begin{array}{c}\text { Entropia } \\
\text { e(di) }\end{array}$ & $\begin{array}{r}\text { Peso } \\
\tilde{\lambda}_{i}\end{array}$ & Média & $\begin{array}{r}\text { Entropia } \\
\text { e(di) }\end{array}$ & $\begin{array}{r}\text { Peso } \\
\tilde{\lambda}_{i}\end{array}$ & Média \\
\hline 6 & 0,9891 & 0,0282 & 4,07 & 0,9939 & 0,0243 & 4,40 \\
7 & 0,9918 & 0,0213 & 3,80 & 0,9936 & 0,0257 & 4,33 \\
8 & 0,9846 & 0,0401 & 3,40 & 0,9936 & 0,0256 & 4,33 \\
9 & 0,9521 & 0,1242 & 2,07 & 0,9801 & 0,0796 & 3,86 \\
10 & 0,9852 & 0,0385 & 3,33 & 0,9944 & 0,0226 & 4,48 \\
11 & 0,9891 & 0,0284 & 3,27 & 0,9929 & 0,0284 & 4,21 \\
12 & 0,9870 & 0,0337 & 3,40 & 0,9906 & 0,0375 & 3,88 \\
13 & 0,9741 & 0,0672 & 3,07 & 0,9879 & 0,0485 & 3,71 \\
14 & 0,9732 & 0,0696 & 2,73 & 0,9701 & 0,1196 & 2,93 \\
15 & 0,9823 & 0,0458 & 2,80 & 0,9580 & 0,1684 & 2,50 \\
16 & 0,9494 & 0,1313 & 2,47 & 0,9739 & 0,1046 & 3,64 \\
17 & 0,9523 & 0,1238 & 2,73 & 0,9762 & 0,0954 & 3,52 \\
18 & 0,9517 & 0,1254 & 2,40 & 0,9747 & 0,1013 & 3,33 \\
19 & 0,9816 & 0,0476 & 3,47 & 0,9903 & 0,0387 & 3,69 \\
20 & 0,9839 & 0,0418 & 3,53 & 0,9923 & 0,0310 & 3,81 \\
21 & 0,9873 & 0,0330 & 3,13 & 0,9878 & 0,0489 & 3,79 \\
\hline Total & & $\mathbf{1}$ & $\mathbf{3 , 1 0}$ & & $\mathbf{1}$ & $\mathbf{3 , 7 8}$ \\
\hline
\end{tabular}

Fonte: Dados de pesquisa

Observa-se na Tabela 2 que a média geral das questões foi de 3,10 para a IES 1 e 3,78 para a IES 2. Com isso, ambas variaram entre o nível "3" (nem concordo/nem discordo) e o 


\section{AVALIAÇÃO DA EFICIÊNCIA DA ESTRUTURA E DO FUNCIONAMENTO DO SISTEMA DE CONTROLE GERENCIAL COM O PERFORMANCE MANAGEMENT AND CONTROL (PMC) EM DUAS UNIVERSIDADES \\ DOI: http://dx.doi.org/10.5007/1983-4535.2018v11n4p57}

patamar "4" (concordo parcialmente), sendo que a menor média $(2,07)$ foi apurada na IES 1 (no caso da questão "9") e a maior média $(4,48)$ coube à IES 2 (no que concerne à pergunta “10”). Quanto à entropia, o menor índice de entropia da informação foi apresentado pela IES 1 na questão "7", com e $\left(\mathrm{d}_{7}\right)$ de 0,9918 e $\tilde{\lambda}_{i}$ de 0,0213 (ou seja, 2,13\%). Por outro lado, o maior índice ocorreu na IES 2 com a pergunta “15”, que obteve e $\left(\mathrm{d}_{15}\right)$ de 0,9580 e peso de 0,1684 (ou 16,84\%).

Adicionalmente, com o fito de facilitar a compreensão dos resultados efetuou-se uma subdivisão em três blocos de questões, onde o bloco "1" abrange as questões relacionadas ao plano estratégico (perguntas "6" a "9"), o bloco " 2 " compreende aquelas vinculadas ao sistema de avaliação de desempenho ("10" a "15") e o terceiro grupo contempla as do controle de gestão ("16" a "21").

No que tange às indagações do primeiro grupo, as questões "6", "7" e "8" apresentaram médias de "4,07”, “3,80” e "3,40” respectivamente no caso IES 1. Por seu turno na IES 2 a questão " 6 " apresentou média de " 4,40 " e nas questões " 7 " e " 8 " a média foi de “4,33". Com isso, concluiu-se que nas duas IES estas situaram-se entre as melhores médias de respostas das questões formuladas no PMC utilizado. No entanto, a outra integrante desse conjunto (pergunta “9”) apresentou resultados divergentes entre as IES. Quanto à IES 1, esta questão obteve a pior média $(2,07 \%)$ e o terceiro pior índice de entropia da informação (peso 0,1242), o que mostra uma dispersão entre os níveis da escala nesse contingente de questões. Por outro lado, na IES 2 tanto a média $(3,81)$ quanto a entropia da informação $(0,0796)$ apresentaram índices medianos, apresentando menor dispersão que a IES 1. É pertinente ressaltar, entretanto, que a característica da IES 1 também se fez presente no estudo de Beuren e Teixeira (2014), onde situação assemelhada foi encontrada.

Em seguida, na avaliação das questões do segundo bloco, em relação a IES 1 observou-se que as questões "10", “11" e "12" apresentaram índices discretos de dispersão $(0,0385 ; 0,0284$ e 0,0337 respectivamente). Entretanto, em relação à IES 2 foi possível dessumir que a questão "10" apresentou a melhor média entre todas as questões relacionadas ao PMC $(4,48)$. Além disso, as respostas da referida questão apresentaram a menor dispersão da estrutura geral da IES (com índice de 0,0226). Por outro lado, as questões "11" e "12" figuraram entre as melhores médias $(4,21$ e 3,88), além de terem índices de dispersão discretos $(0,0284$ e 0,0375$)$. 


\section{AVALIAÇÃO DA EFICIÊNCIA DA ESTRUTURA E DO FUNCIONAMENTO DO SISTEMA DE CONTROLE GERENCIAL COM O PERFORMANCE MANAGEMENT AND CONTROL (PMC) EM DUAS UNIVERSIDADES \\ DOI: http://dx.doi.org/10.5007/1983-4535.2018v11n4p57}

Em sentido oposto, este segundo bloco concentrou as duas questões com menor média: a pergunta "15" (com 2,50) e a pergunta "14" (com 2,93). Adicionalmente, estas duas apresentaram altos índices de dispersão, com 0,1684 e 0,1196 respectivamente. Entretanto, os valores apurados para a IES 1 revelaram índices medianos de dispersão $(0,0458$ e 0,0696$)$ e de média $(2,80$ e 2,73$)$ para as referidas questões. Ainda, para a outra participante deste bloco (questão “13”) foi calculada média de 3,07 e 3,71 e um índice de dispersão mediano (0,0672 e $0,0485)$ para a IES 1 e IES 2, respectivamente.

Esses resultados indicam que as duas universidades em tela não possuem um sistema de avaliação de desempenho consolidado, pois ambas apresentaram consideráveis índices de dispersão, gerando questionamentos em relação a sua eficácia. Esses resultados também se assemelham aos "achados" da entidade pesquisada por Beuren e Ferreira (2014), especialmente no que concerne à questão " 15 " da IES 2 , de vez que nesta e naquela pesquisa tal pergunta se apresentou com o pior índice de dispersão entre as 16 questões da estrutura do PMC utilizada.

Com relação às questões do bloco 3 (itens "16" a "21") foi possível notar situação semelhante entre as duas entidades, especialmente no que tange às questões "16", "17" e “18”, pois as mesmas concentraram altos índices de dispersão e baixas médias. Na IES 1 as referidas indagações apresentaram o pior $(0,1313)$, o quarto $(0,1238)$ e o segundo $(0,1254)$ piores índices de dispersão das 16 questões aplicadas especificamente sobre o PMC. Ainda, tais questões também tiveram as menores médias do conjunto de questões (com "2,47" para a primeira, "2,73” para a segunda e "2,40” para última). Na IES 2 os índices de dispersão apresentados posicionam-se como o terceiro, o quinto e o quarto piores índices das 16 questões do PMC, com peso de "0,1046", “0,0954” e “0,1013”. No que concerne às médias respectivas (“3,64”, “3,52” e “3,33”), estas figuraram entre as piores médias do bloco e entre as mais baixas do conjunto das 16 questões aplicadas.

Notou-se também que as respostas da pergunta " 20 " foram as que apresentaram o melhor resultado entre as seis indagações efetuadas, com média de 3,53 na IES 1 (ocupando o terceiro lugar entre o conjunto total de questões) e média de 3,81 para a IES 2 (classificada em oitavo lugar entre o conjunto total de questões). Quanto às questões "19" e "21", constatou-se uma inversão nos resultados das duas instituições. Enquanto que a IES 1 apresentou entropia mediana $(0,0476)$ para a questão “ 19 ” e discreta $(0,0330)$ para a questão 


\section{AVALIAÇÃO DA EFICIÊNCIA DA ESTRUTURA E DO FUNCIONAMENTO DO SISTEMA DE CONTROLE GERENCIAL COM O PERFORMANCE MANAGEMENT AND CONTROL (PMC) EM DUAS UNIVERSIDADES \\ DOI: http://dx.doi.org/10.5007/1983-4535.2018v11n4p57}

“21", a IES 2 teve resultado discreto $(0,0387)$ para a questão "19" e mediano $(0,0489)$ para a questão “21”.

Os resultados deste último bloco convergem parcialmente com os "achados" de Beuren e Teixeira (2014). Ou seja, neste estudo o referido bloco de questões foi o que mais concentrou dispersão nos resultados e teve os níveis mais elevados de entropia, enquanto que nos resultados mensurados por Beuren e Teixeira (2014) os índices a respeito foram um pouco menores.

\section{CONCLUSÕES DO ESTUDO}

Este estudo teve o objetivo de conhecer a opinião dos coordenadores de cursos sobre a utilização dos sistemas de controle gerencial (SCG) para avaliação de desempenho em duas IES comunitárias situadas no oeste de Santa Catarina, por intermédio do PMC. Nesse rumo, pretendia buscar respostas para dúvidas relacionadas à visão desses gestores sobre essa utilização.

A partir das respostas colhidas por intermédio de um questionário foi possível concluir que, em relação às perguntas acerca de aspectos do planejamento estratégico, os coordenadores declararam conhecer a missão, visão e os objetivos estratégicos das duas universidades, bem como conhecer as atividades e processos necessários para implementação da estratégia adotada. No entanto, o resultado da questão "9" indicou a existência de uma falha na comunicação e divulgação do plano estratégico aos coordenadores. Isso ocorreu especialmente na IES 1 , pois $40 \%$ dos participantes afirmaram não ter recebido (por escrito) o referido plano e outros $46,67 \%$ discordaram parcialmente ou mostraram-se indiferentes ao assunto. Ainda, houve alto índice de dispersão nas respostas a respeito $(0,1242)$. No âmbito da IES 2, esta apresentou percentuais menos acentuados, porém cerca de $28,5 \%$ dos respondentes também se mostram insatisfeitos em relação a esse quesito, o que se refletiu também num nível indesejado de entropia $(0,0796)$.

No que tange às questões que investigaram sobre o sistema de avaliação de desempenho (perguntas “10" a "15"), os resultados evidenciaram divergências entre os contextos das duas entidades pesquisadas. Quando indagados se a estrutura existente permite adotar o sistema de avaliação dos resultados, apenas 40\% dos respondentes da IES 1 concordaram (ainda que de forma parcial), enquanto que a performance da IES 2 nesse quesito foi de $90,48 \%$ de respostas positivas. Ainda, a maioria dos respondentes $(85,71 \%)$ 


\section{AVALIAÇÃO DA EFICIÊNCIA DA ESTRUTURA E DO FUNCIONAMENTO DO SISTEMA DE CONTROLE GERENCIAL COM O PERFORMANCE MANAGEMENT AND CONTROL (PMC) EM DUAS UNIVERSIDADES \\ DOI: http://dx.doi.org/10.5007/1983-4535.2018v11n4p57}

concordaram que a IES 2 utiliza indicadores-chave de desempenho ligados aos objetivos estratégicos. Todavia, na IES 1 foi apurado que somente $46,67 \%$ dos coordenadores concordaram a respeito desse ponto.

Quando questionados se as metas fixadas para avaliar o desempenho são adequadas para permitir que os objetivos estratégicos sejam alcançados, os respondentes mostraram-se majoritariamente favoráveis acerca disso, de vez que 53,33\% (na IES 1) e 78,57\% (na IES 2) dos respondentes asseveraram que concordam totalmente ou parcialmente sobre o tema.

No que concerne à eficiência do processo de avaliação existente, as respostas apresentaram dispersão mediana em relação ao conjunto de 16 questões do PMC. No entanto, por se tratar de um quesito tão importante pode-se considerar que a questão apresentou dispersão indesejada nos resultados nas duas entidades, assim como ocorreu em relação às recompensas e penalizações propostas na estrutura de avaliação existente. Com isso, os resultados apontaram que os coordenadores não têm acesso ou não consideram justas as formas de recompensas ou penalizações quando do alcance (ou não) dos objetivos e metas propostos.

Em relação às questões do sistema de avaliação de desempenho (itens "16" a "21"), confirma-se a falha na comunicação observada na pergunta "9", pois as indagações referentes ao recebimento de informações por parte dos coordenadores (questões "16" a "18") situaramse entre os maiores índices de dispersão dos resultados (“0,1313”, “0,1238” e "0,1254” na IES 1 e "0,1046", “0,0954” e "0,1013" na IES 2, respectivamente) e entre as menores médias (“2,47”, “2,73” e "2,40” na IES 1 e “3,64”, “3,52” e “3,33” na IES 2, de forma respectiva). Em virtude disso, entendeu-se coerente dessumir que o controle de gestão não foi percebido pelos gestores como eficaz, mesmo que os envolvidos conheçam os objetivos, o plano estratégico e a forma de execução e implementação destes. Deste modo, restou evidenciado que desconhecem ou não têm acesso às informações que permitem acompanhar a avaliação e o desempenho, tanto dos cursos como da função de coordenador que exercem.

Os resultados, de modo geral, se assemelham aos "achados" de Ferreira e Otley (2006) e Beuren e Teixeira (2014), especialmente ao que se refere aos resultados da IES 2. Em relação à análise descritiva do primeiro bloco de questões se verificou que os resultados da IES 2 são assemelhados aos de Beuren e Teixeira (2014), enquanto que divergem dos achados da IES 1. Contudo, a análise descritiva do segundo bloco de questões indica semelhança dos resultados das duas entidades em tela com os estudos utilizados como referência 


\section{AVALIAÇÃO DA EFICIÊNCIA DA ESTRUTURA E DO FUNCIONAMENTO DO SISTEMA DE CONTROLE GERENCIAL COM O PERFORMANCE MANAGEMENT AND CONTROL (PMC) EM DUAS UNIVERSIDADES \\ DOI: http://dx.doi.org/10.5007/1983-4535.2018v11n4p57}

(FERREIRA; OTLEY, 2006; BEUREN; TEIXEIRA, 2014). Isso também se aplica aos resultados da análise descritiva relativa aos controles de gestão do desempenho, os quais se coadunaram com os encontrados por Ferreira e Otley (2006) e Beuren e Teixeira (2014).

Cabe ressaltar também que em relação à análise da entropia da informação os resultados desta pesquisa apresentam-se em parte convergentes e parcialmente discrepantes do estudo de Beuren e Teixeira (2014). A análise das questões relacionadas ao plano estratégico indicou semelhança nos resultados da IES 1. Entretanto, no que tange à avaliação do bloco 2 os "achados" de Beuren e Teixeira (2014) convergem com os identificados à IES 2 , especialmente na questão " 15 ", de vez que as duas entidades pesquisadas tiveram nesta questão o maior índice de entropia, indicando maior dispersão entre as 16 questões propostas. No tocante aos resultados do último conjunto de questões, estes convergem parcialmente com a pesquisa de Beuren e Teixeira (2014) porque neste estudo o referido bloco de questões foi aquele que mais concentrou dispersão nos resultados e teve os níveis mais elevados de entropia para ambas as instituições educacionais visadas, enquanto que nos resultados mensurados por Beuren e Teixeira (2014) os índices a respeito foram menos elevados.

Quanto às limitações que devem ser consideradas, cabe mencionar dois aspectos principais. O primeiro refere-se ao percentual de respondentes da pesquisa, que abrangeu somente $34,74 \%$ dos possíveis participantes no caso da IES 1 e cerca de 40,38\% no âmbito da IES 2. Com isso, os achados de pesquisa devem ser considerados como circunscritos às duas instituições, visto que este nível de abrangência não permite generalizações. Adicionalmente, as pesquisas do tipo survey estão sujeitas a limitações, que podem advir da existência de potenciais vieses de não respondentes, de aquiescência e de respostas socialmente desejáveis. Ou seja, uma das possíveis limitações desse tipo de estudo é a ênfase em aspectos perceptivos, visto que levantamentos recolhem dados que se referem às percepções das pessoas. Isto pode gerar distorções, porque há muita divergência entre o que as pessoas efetivamente fazem (ou sentem) e o que elas revelam a respeito.

Como sugestão para estudos futuros se recomenda a aplicação deste estudo em outras instituições de ensino superior como forma de permitir a comparabilidade de diversos estudos, bem como contribuir para o aprofundamento do tema. 


\section{REFERÊNCIAS}

AGUIAR, A. B. de; FREZATTI, F. Escolha da estrutura apropriada de um sistema de controle gerencial: uma proposta de análise. Revista de Educação e Pesquisa em Contabilidade, v.1, n.3, p.21-44, 2007.

ANTHONY, R.N.; GOVINDARAJAN, V. Sistemas de controle gerencial. São Paulo: Atlas, 2006.

BEUREN, I.M.; TEIXEIRA, S.A. Avaliação dos sistemas de controle gerencial em instituição de ensino superior com o Performance Management and Control. Revista de Gestão da Tecnologia e Sistemas de Informação, v.11, p.169-192, 2014.

BEUREN, I. M.; KLEIN, L.; LARA, F. L.; ALMEIDA, L. B. de. Percepção de justiça nos sistemas de controle gerencial aumenta comprometimento e confiança dos gestores? Revista de Administração Contemporânea, v. 20, n. 2, art. 5, p. 216-237, mar./abr., 2016.

BEUREN, I. M.; ORO, I. M. Relação entre estratégia de diferenciação e inovação e sistemas de controle gerencial. Revista de Administração Contemporânea, v. 18, n. 3, p. 285, 2014.

BEUREN, I. M.; SANTANA, S. V.; THEIS, M. B. A inter-relação entre os sistemas de controle gerencial e as estratégias organizacionais: um estudo de caso. Race: Revista de Administração, Contabilidade e Economia, v. 13, n. 3, p. 919-954, 2014.

BORTOLUZZI, S. C; ENSSLIN, S. R; ENSSLIN, L. Importância e ferramentas de avaliação de desempenho para redes de pequenas e médias empresas: análise crítica da literatura nacional versus internacional. Revista Produção Online, v. 15, n. 4, p. 1148-1173, 2015.

CHENHALL, R. H. Management control systems design within its organizational context: findings from contingency-based research and directions for the future. Accounting, Organizations and Society, v. 28, n. 2, p. 127-168, 2003.

DAVILA, A.; FOSTER, G.; LI, M. Reasons for management control systems adoption: Insights from product development systems choice by early-stage entrepreneurial companies. Accounting, Organizations and Society, v. 34, n. 3, p. 322-347, 2009.

DAL MAGRO, C. B; GORLA, M. C; LAVARDA, C. E. F. Sistema de controle de desempenho como um pacote: um estudo em empresas nacionais da região sul. In: XXII CONGRESSO BRASILEIRO DE CUSTOS - CBC, 2016, Porto de Galinhas. Anais...São Leopoldo, 2016.

DRURY, C. Management and cost accounting. 6 ed. London: Thomson Learning, 2004.

DUTRA, A. Metodologia para avaliar e aperfeiçoar o desempenho organizacional: incorporando a dimensão integrativa à MCDA Construtivista-Sistêmico-Sinergética. Tese (Doutorado em Engenharia de Produção) - Universidade Federal de Santa Catarina, Florianópolis, 2003. 
FERREIRA, A.; OTLEY, D. The design and use of performance management systems: an extended framework for analisys. Management Accouting Research, v.20, p.263-282, 2006.

GOMES, J.S.; SALAS, J.M. Controle de gestão: uma abordagem contextual e organizacional. São Paulo: Atlas, 2001.

HAMPTON, D. R. Administração: processos administrativos. São Paulo: MacGraw-Hill, 1990.

HOPWOOD, A. G. An empirical study of the role of accounting data in performance evaluation. Journal of Accounting Research, p. 156-182, 1972.

JACOMOSSI, F.A.; SILVA, M. Z. da. Influência da incerteza ambiental na utilização de sistemas de controle gerencial em uma instituição de ensino superior. REGE - Revista de Gestão, n.23, p. 75-85, 2016.

MERCHANT, K. A.; VAN DER STEDE, W. A. Management control systems: performance measurement, evaluation and incentives. Pearson Education, 2007.

MOSIMANN, C.P.; FISCH, S. Controladoria. 2a ed. São Paulo: Atlas, 1999.

NISIYAMA, E. K.; OYODAMARI, J. C. T.; YEN-TSANG, C.; AGUIAR, A. B. de. O uso dos sistemas de controle gerencial e técnicas de gestão operacional. BBR Brazilian Business Review, v. 13, n. 2, art. 3, p 57-83, mar./abr. 2016.

OTLEY, D. Performance management: a framework for management control systems research. Management Accounting Research, v. 10, n. 4, p. 363-382, 1999.

PASSOS, C. A.; SPERS, R. G. Modelo de avaliação de desempenho organizacional para pequenas e médias empresas. Revista Ibero-Americana de Estratégia, v. 13, n. 4, p. 44-58, 2014.

RAUPP, F.M; MARTINS, S.J.; BEUREN, I.M. Utilização de controles de gestão nas maiores indústrias catarinense. Revista Controladoria e Finanças - USP, n. 40, p. 120 - 132, jan./abr., 2006.

REIS NETO, M.T.; MARQUES, A.L. A remuneração variável e sua contribuição para a melhoria da gestão. Revista de Negócios, v.9, n.1, p. 05-17, 2014.

RICHARDSON, R. J. Pesquisa social: métodos e técnicas. $3^{\text {a }}$ ed. São Paulo: atlas, 2014.

ROCHA, I. Grau de entropia da informação em indicadores econômico-financeiros das empresas que participam dos níveis de governança corporativa da BM\&FBovespa. 2010. 155 p. Dissertação (Mestrado em Ciências Contábeis) - Universidade Regional de Blumenau, Blumenau, 2010. 
ROESCH, S. M. A. Projetos de estágio e de pesquisa em administração: guia para estágios, trabalhos de conclusão, dissertações e estudos de caso. 3. ed. 2. reimpr. São Paulo: São Paulo, Atlas, 2006.

SANTOS, L.; BRONNEMANN, M. R. Desafios da gestão em instituições de ensino superior: um estudo de caso a partir da percepção de diretores de centro de uma IES pública do sul do Brasil. Revista Gestão Universitária na América Latina - GUAL, v. 6, n. 1, p. 01-21, 2013.

SEMESP - Sindicato das Entidades Mantenedoras de Estabelecimentos de Ensino Superior no Estado de São Paulo. Mapa do Ensino Superior no Brasil. São Paulo: SEMESP, 2015.

SEVERINO, A. J. Metodologia do trabalho científico. 23. ed. rev. São Paulo: Cortez, 2007. SILVA, A. C. R. Metodologia da pesquisa aplicada à contabilidade: orientações de estudos, projetos, relatórios, monografias, dissertações, teses. São Paulo: Atlas, 2003.

SIMONS, R. Levers of control: how managers use innovative control systems to drive strategic renewal. Boston: Harvard Business School Press, 1995.

TACHIZAWA, T.; ANDRADE, R. O. B. Gestão de instituição de ensino. Rio de Janeiro: FGV, 2002.

ZELENY, M. Multiple criteria decision making. New York: McGraw-Hill, 1982. 